J3eA, Journal sur l'enseignement des sciences et technologies de l'information et des systèmes, Volume 4, Hors-Série 2, 18 (2005)

DOI : http://dx.doi.org/10.1051/bib-j3ea:2005718

(C) EDP Sciences, 2005

Circuits électroniques visualisés par vidéomodèles

\author{
F. Gueuning
}

Unité Électronique

Institut Supérieur Industriel ECAM

14 rue du Tir

B-1060 Bruxelles, Belgique 


\title{
CIRCUITS ÉLECTRONIQUES VISUALISÉS PAR VIDÉOMODÈLES
}

\author{
F. Gueuning, Unité Électronique, Institut Supérieur Industriel ECAM, 14, rue du Tir, \\ B-1060 Bruxelles.gng@ecam.be
}

\section{Résumé :}

Les vidéomodèles se révèlent particulièrement efficaces pour montrer les circuits électroniques en pleine action. L'objectif est de le montrer par la pratique. On utilise les vidéomodèles pour représenter des circuits électroniques à diodes, transistors bipolaires et amplificateur opérationnel. Après une présentation des symboles 3D de ces composants, on montre un amplificateur à un transistor en émetteur commun, un redresseur à amplificateur opérationnel, un circuit intégré à 20 transistors et un circuit RLC sur lequel on propose également une représentation de la puissance instantanée transmise d'un dipôle à l'autre. Grâce aux vidéomodèles, tous ces circuits sont nettement mieux perçus par la plupart des étudiants .

Mots clés : vidéomodèle, animation 3D, circuit, électronique, simulation, comportement dynamique, modèle 3D.

\section{INTRODUCTION}

Les vidéomodèles constituent en quelques sortes le cinéma des circuits dont les acteurs sont les composants qui interagissent entre eux [1]. Si de nouveaux acteurs doivent intervenir, il faut d'abord les créer et s'assurer de la qualité de leur expression. On propose ici les représentations 3D de trois composants principaux de l'électronique: la diode, le transistor bipolaire, et l'amplificateur opérationnel.

On exploite ensuite ces composants, qui s'ajoutent à ceux de [1], dans plusieurs circuits afin de montrer des secteurs importants d'applications des vidéomodèles. Ces exemples mettent en évidence la faculté des vidéomodèles d'intégrer le fonctionnement d'ensemble et les phénomènes de second ordre sans nuire à la lisibilité.

\section{CRÉATION DE COMPOSANTS}

La création de symboles 3D de composants n'est pas un travail trivial. Pour que les vidéomodèles ne se démodent pas, il faut les réaliser avec des symboles bien pensés dès le départ. Ceux-ci doivent être simples et généralistes pour convenir dans des circuits très divers, mais également être expressifs pour permettre au composant de dévoiler son rôle précis dans chaque situation. Le symbole doit être utilisable tant pour rendre le fonctionnement d"ensemble que celui du détail. Pour être immédiatement reconnu, il doit rappeler le symbole 2D conventionnel. Il est également important qu'il mette en évidence les tensions entre bornes du composant.

\subsection{Symboles des transistors NPN et PNP}

Pour respecter les objectifs cités ci-dessus, la création du symbole du transistor bipolaire a demandé plusieurs mois pour aboutir. Puisqu'en électronique le rouge est la couleur du positif tandis que le bleu est la couleur du négatif, on propose, de manière générale, de représenter en bleu les composants où le courant d'électrons domine, et en rouge ceux où le courant de trous domine. On propose donc de représenter le transistor NPN en bleu et le transistor PNP en rouge.

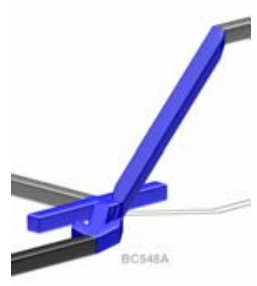

(a) bloqué

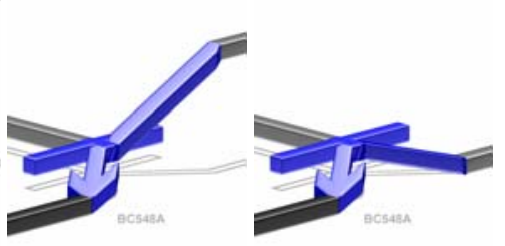

(b) actif (c) saturé 


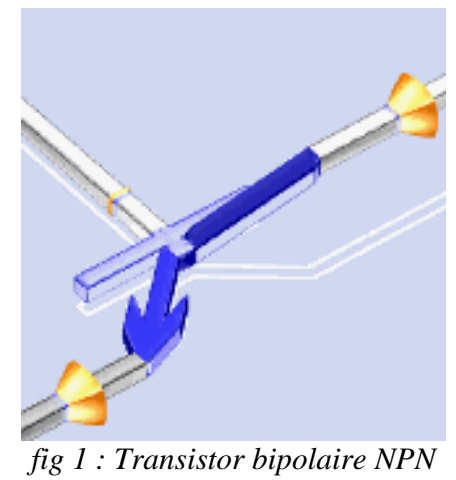

Le symbole est composé (fig 1 et 2), au potentiel de la base, d'un T à barre centrale presque inexistante d'où partent respectivement vers l'émetteur et le collecteur une flèche et une barre obliques. Le sens de la flèche indique, comme sur le symbole 2D universel, s'il s'agit d'un transistor NPN ou PNP. Puisque les tensions sont visibles en permanence, la représentation $3 \mathrm{D}$ permet de voir le régime du transistor, ce qui est très intéressant dans des circuits complexes. Notons que l'idée d'allonger les bras du transistor en fonction de la tension est déjà présente dans [2] mais on s'y contente de simples lignes pour la représentation.
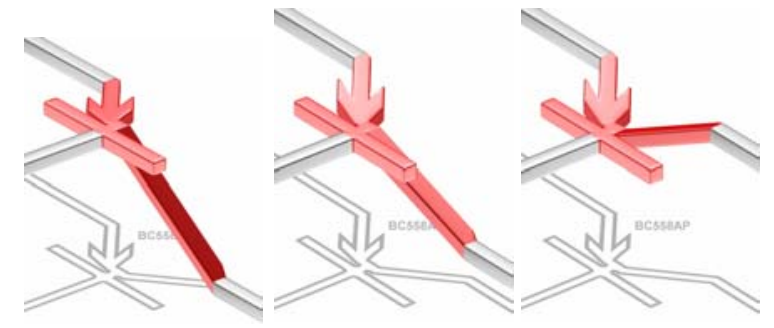

(a) bloqué (b) actif

(c) saturé

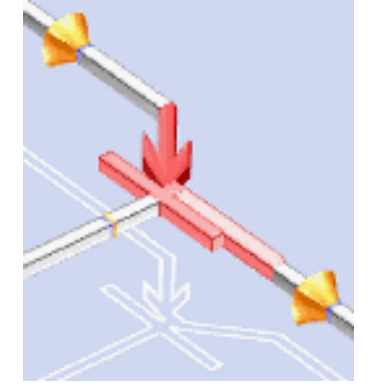

fig 2 : Transistor bipolaire PNP

Sur les figures 1.a et 2.a, le régime bloqué se déduit de ce que la tension émetteur-base est trop faible pour rendre la jonction passante. Lorsque cette tension devient suffisante, et bien sûr de sens adéquat, le transistor passe en régime actif (fig. 1.b et 2.b). Plus le transistor est passant et plus sa tension collecteur-émetteur tend à diminuer (en valeur absolue). Lorsqu'elle devient proche du court-circuit, le transistor est saturé (fig. 1.c et 2.c).

\subsection{Symbole de la diode}

La diode est représentée à l'aide du symbole de la figure 3. On reconnaît la diode passante au fait que la flèche pointe vers le bas avec une tension suffisante qui devient alors peu dépendante du courant (circulant évidemment en sens passant).

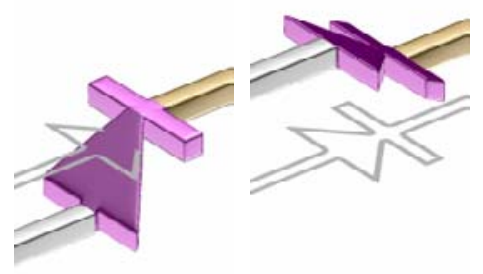

(a) bloquée $\quad$ (b) passante fig 3 : Diode classique 
Les coordonnées $\mathrm{X}$ et $\mathrm{Y}$ de chaque point du symbole restent toujours constantes. En sens passant, la coordonnée $\mathrm{Z}$ évolue linéairement entre les deux bornes (où elle correspond quantitativement au potentiel). En sens bloquant, la barre de cathode est maintenue horizontale mais le triangle évolue linéairement jusqu'à elle.

Dans [3] où la diode est faite de simples lignes, lorsque la diode est bloquée, le triangle reste horizontal et se détache de la barre de cathode qui a son propre potentiel. L'avantage est de constater un circuit ouvert, l'inconvénient est qu'il est plus difficile d'identifier le composant dans un circuit complexe.

\subsection{Symbole de l'amplificateur opérationnel}

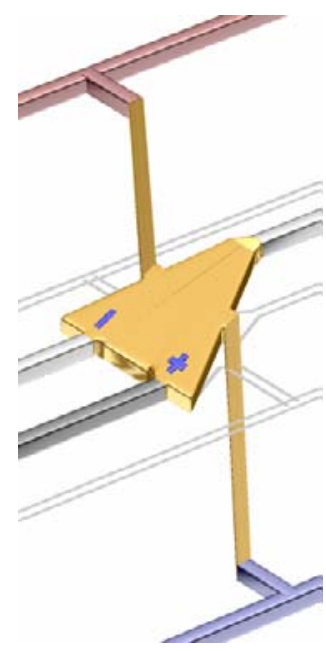

Le symbole de l'amlificateur opérationnel (Aop) est constitué de six zones plates : correspondant (fig.4)

- aux entrées inverseuse et non inverseuse

- à un triangle entre elles

- à la sortie

- aux deux bornes d'alimentation.

Ces dernières font mieux percevoir la saturation de l'Aop souvent oubliées par les débutants. Pour ne pas alourdir le dessin, il est fréquent qu'elles n'apparaissent pas (fig. 8).

Tant que la tension différentielle d'entrée est proche de $0 \mathrm{~V}$, les trois premières surfaces du symbole sont dans un même plan (fig. 8 en bas). Ce n'est plus le cas si la tension différentielle devient plus importante, ce qui traduit un gain dégradé de l'Aop (fig. 8 en haut).

La zone de sortie est actuellement maintenue horizontale en permanence. Peut-être que dans le futur, on lui fera jouer un rôle (ex: indiquer la tension sur l'impédance interne de sortie de l'Aop).

fig 4 : Aop et ses connexions d'alimentations

\section{EXEMPLES DE CIRCUITS MIEUX PERÇUS PAR VIDÉOMODÈLES}

\subsection{Transistor PNP monté en émetteur commun}

Le schéma de la figure 5 représente un amplificateur à un transistor PNP monté en émetteur commun. Le vidéomodèle correspondant, dont une vue est donnée à la figure 6 , est montré au tout début du cours d'introduction à l'électronique en $2^{\mathrm{e}}$ année à l'ECAM.

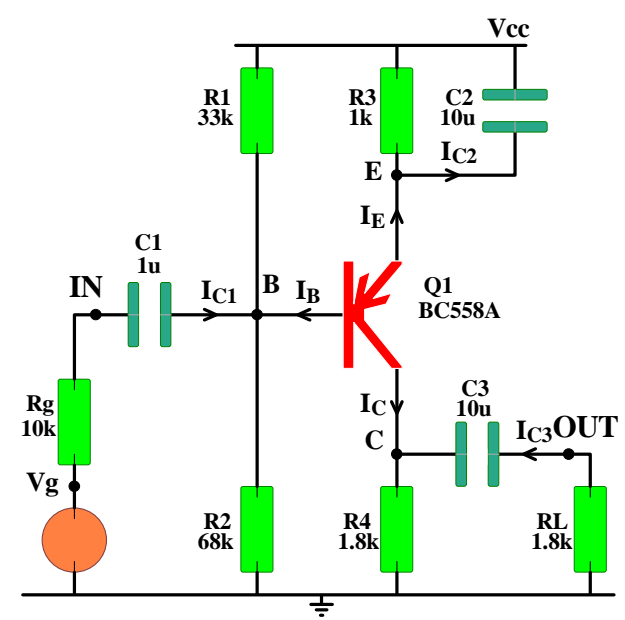

fig 5 : Schéma traditionnel (émetteur commun)

On commence par constater l'existence d'un dipôle de Thévenin (à gauche) produisant un faible signal sinusoïdal, et d'une charge (à droite) où le signal est déphasé et amplifié, le quadripôle central joue donc le rôle d'amplificateur mais on ignore à ce stade son mécanisme d'amplification. On observe également le rôle des condensateurs $\mathrm{C} 1$ et $\mathrm{C} 3$ aptes à transmettre les variations de potentiel tout en reprenant sur eux la tension continue, ainsi que le rôle de $\mathrm{C} 2$ qui neutralise les variations du potentiel $\mathrm{V}_{\mathrm{E}}$, ce qui fait de $\mathrm{E}$ une masse d'un point de vue variationnel. On constate également le rôle des résistances de polarisation destinées à suspendre le transistor quelque part entre les rails d'alimentation. On observe finalement le lien entre les courants $\mathrm{I}_{\mathrm{B}}$ et $\mathrm{I}_{\mathrm{C}} \mathrm{du}$ 
transistor et donc son rôle de "vanne" commandée en courant. L'image comporte un zoom sur le transistor où l'on voit mieux les courants $\mathrm{I}_{\mathrm{B}}$ et $\mathrm{I}_{\mathrm{E}}$. Compte tenu de leurs valeurs respectives de $18.2 \mu \mathrm{A}$ et $3.33 \mathrm{~mA}$, et afin que les cônes correspondants restent visibles, l'amplitude de ces derniers est proportionnelle à $\mathrm{I}^{0.4}$ plutôt qu'à $\mathrm{I}$, comme mentionné en [1].
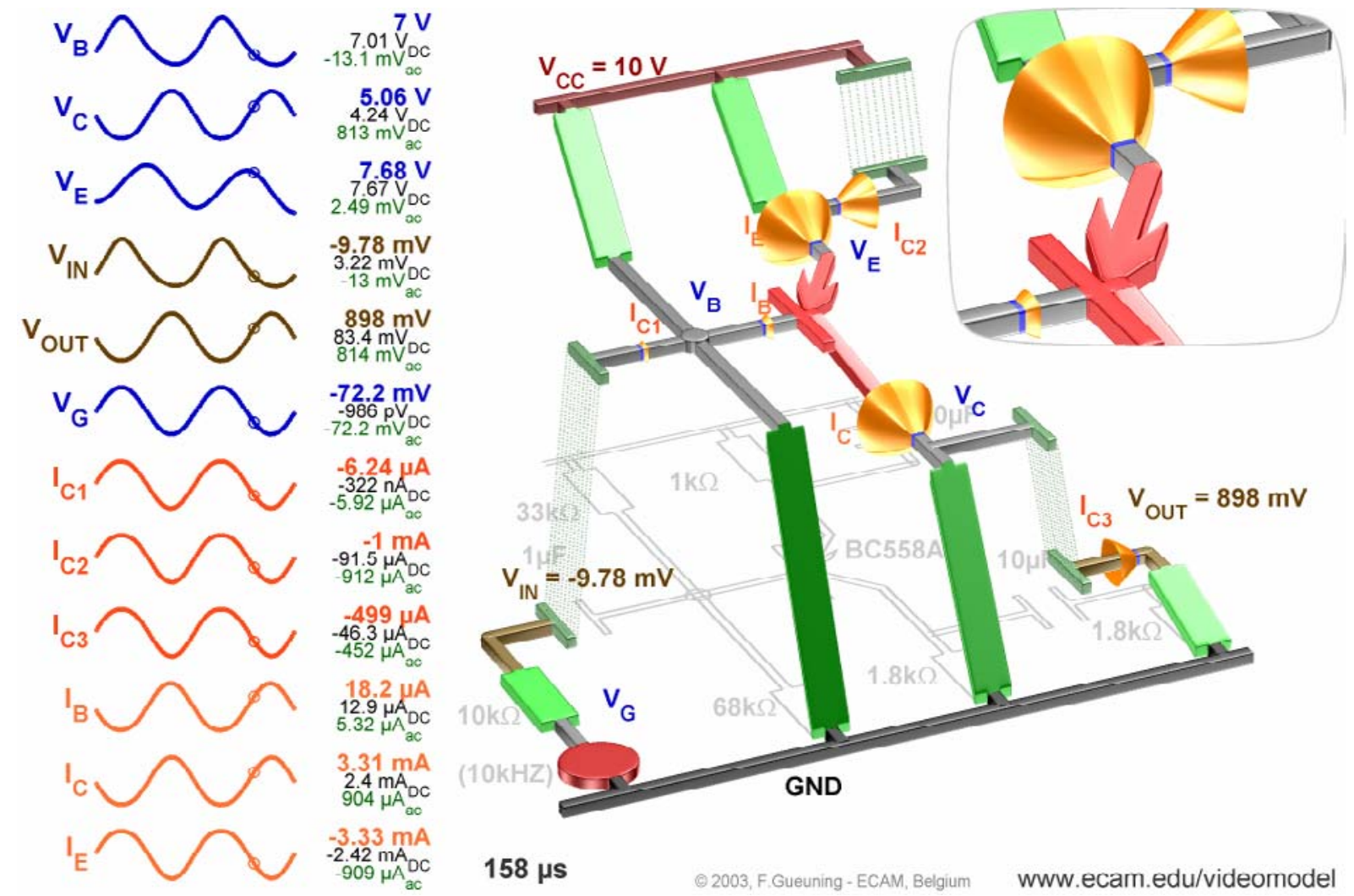

fig 6: Image 79 du vidéomodèle qui en compte 100 (amplificateur à transistor PNP en émetteur commun)

La couleur de fond des courbes indique la fréquence des signaux sinusoïdaux, ce qui permet de rendre compte du comportement fréquentiel du circuit. Le chiffre correspondant à la couleur est le logarithme en base 10 de la fréquence. $100 \mathrm{~Hz} \quad 1 \mathrm{kHz} \quad 10 \mathrm{kHz} \quad 100 \mathrm{kHz} \quad 1 \mathrm{MHz}$

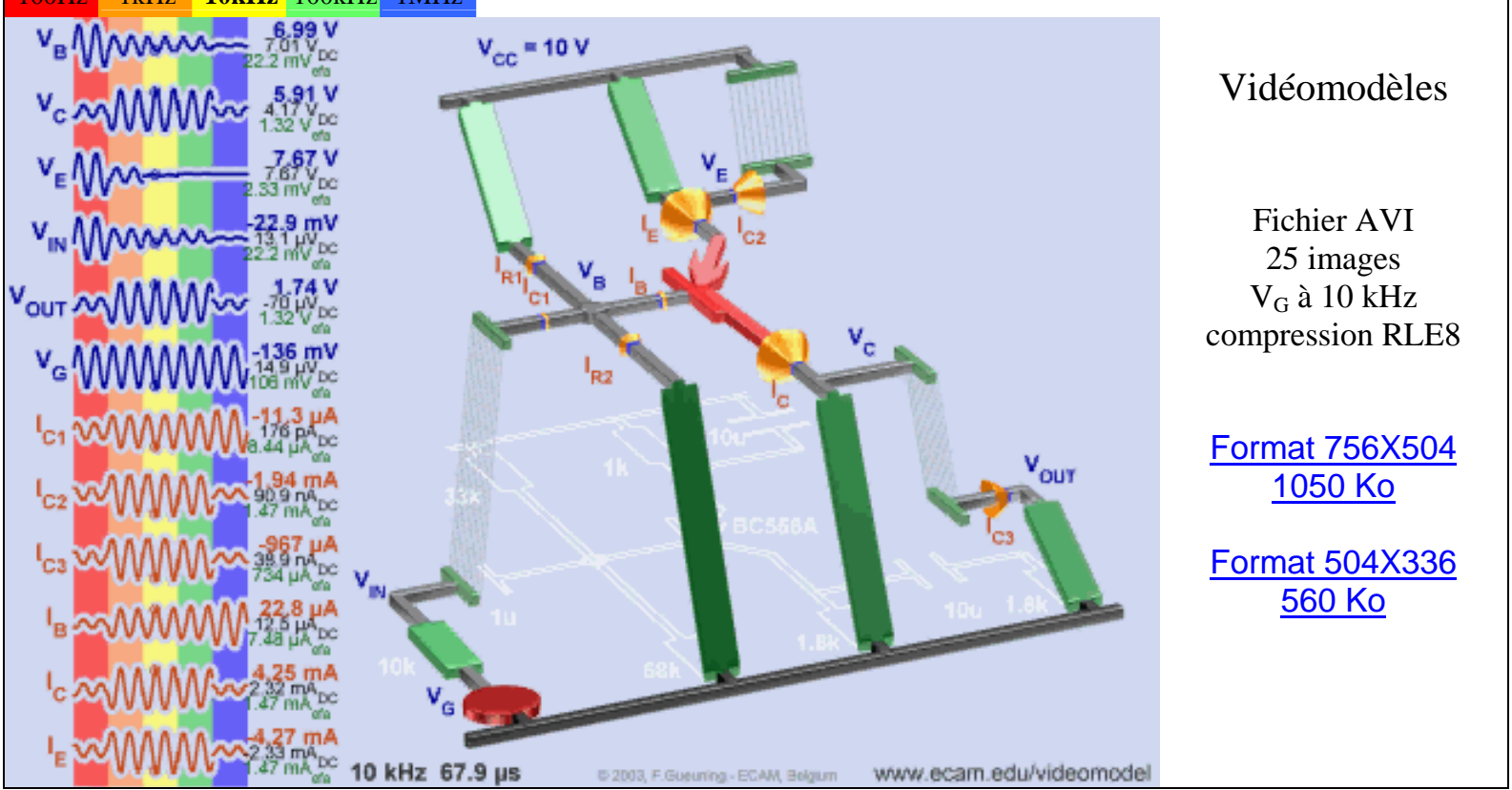

Toutes ces observations effectuées dès le départ sur un même vidéomodèle permettent de dégager les étapes de l'étude d'un tel circuit : lois de base de l'électricité, comportement du transistor, point de fonctionnement, petits signaux, performances globales. 
Par la suite, on peut comparer différentes variantes du circuit dont les vidéomodèles ont été réalisés.

Notons (fig. 6) que, les résistances, pourtant de couleur identique a priori, ne présentent pas la même luminance à cause des réflexions spéculaires de l'éclairage virtuel sur les surfaces des symboles. Cette différence est peu gênante par le fait que la couleur permet d'identifier immédiatement les composants.

\subsection{Redresseur à amplificateur opérationnel}

Ce circuit permet de montrer l'Aop en comportement linéaire (rétroaction négative) ou saturé ainsi que les phénomènes survenant lors du passage de l'un à l'autre. On donne son schéma (fig.7) et deux vues extraites du vidéomodèle correspondant (fig.8).

- Comportement linéaire

Si la diode est passante (fig.8 en bas), le courant peut circuler entre la sortie de l'Aop et la source de tension, ce qui provoque des chutes de tension dans la diode et dans le diviseur résistif. L'Aop ajuste sa sortie pour que sa tension différentielle d'entrée soit nulle, ce qui conduit, dans le cas présent où les deux résistances sont identiques, à $\mathrm{V}_{\text {OUT }}=-\mathrm{V}_{\mathrm{IN}}$.

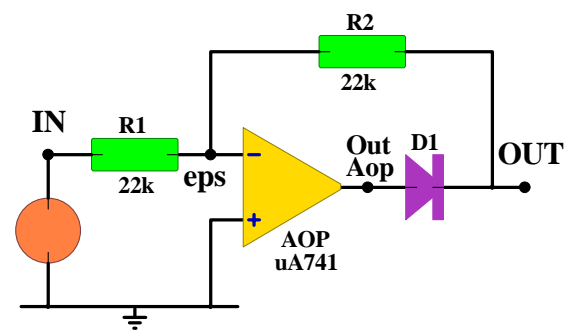

fig 7 : Redresseur à amplificateur opérationnel

- Disparition de la boucle de rétroaction

Si la tension d'entrée devient positive, la tension de sortie de l'Aop tend à s'inverser, ce qui bloque la diode et annule le courant et donc les chutes de tension sur les résistances. $\mathrm{V}_{\mathrm{OUT}}=+\mathrm{V}_{\mathrm{IN}}$ et l'Aop part en saturation.

- De la saturation au mode linéaire

Sur la figure 8 en haut, $V_{\text {IN }}$ est déjà redevenue négative, l'amplificateur revient de la saturation négative, ce qui lui prend un certain temps durant lequel la diode est encore bloquée, les chutes de tension sur les résistances sont donc nulles et, encore pendant un court instant, $\mathrm{V}_{\text {out }}=+\mathrm{V}_{\mathrm{IN}}$ comme durant toute la phase de saturation. Notons que la simulation tient compte de capacités parasites : on voit qu'à cet instant : $V_{\text {IN }}=-375 m V$ et $V_{\text {OUT }}=-348 m V$

Tous ces phénomènes sont bien plus simples à assimiler si on les voit se dérouler dans le vidéomodèle que si on ne dispose pas d'outil de visualisation dynamique, comme c'est malheureusement le cas dans le présent texte. 


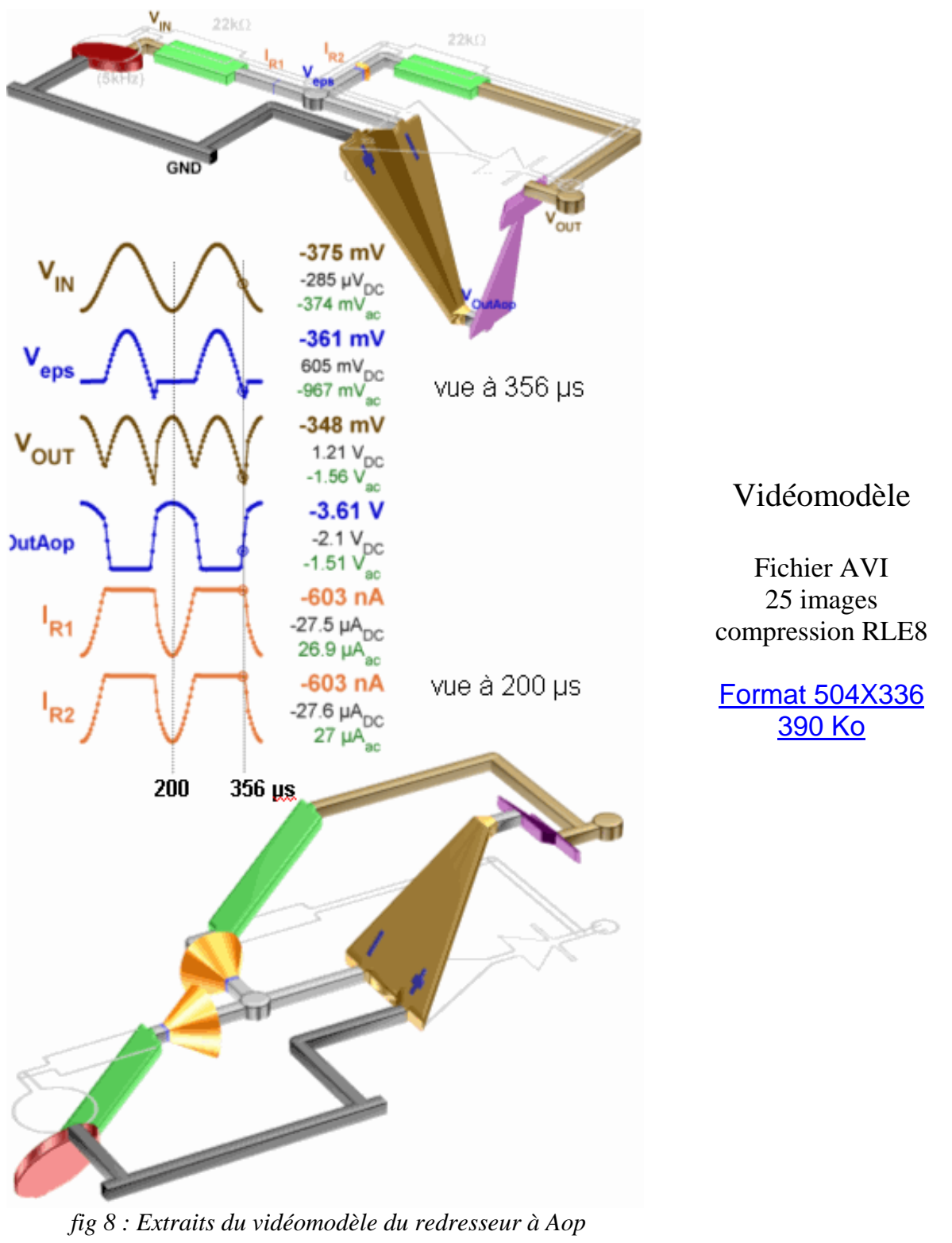

\subsection{Autres circuits montrés aux étudiants}

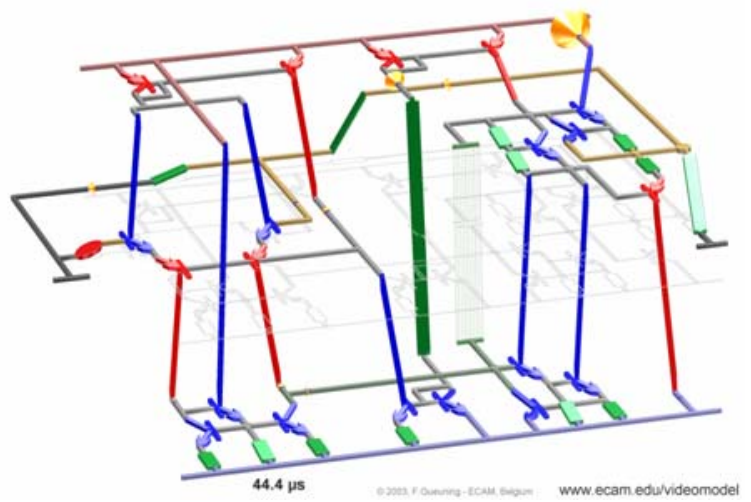

fig 9 : Circuit intégré $\mu$ A741 en montage non inverseur

D'autres circuits électriques ou électroniques sont également montrés aux étudiants tout au long des cours de $2^{\mathrm{e}}$ et $3^{\mathrm{e}}$ année. Suivant l'état d'avancement du cours, le même vidéomodèle peut servir pour une étude de base ou une étude approfondie avec calcul des équations principales et vérification des ordres de grandeur. Citons par 
exemple l'étude du fonctionnement interne d'un circuit intégré, l'Aop $\mu$ A741 en montage non inverseur (fig. 9) ou encore l'analyse d'un circuit RLC série (fig. 10).

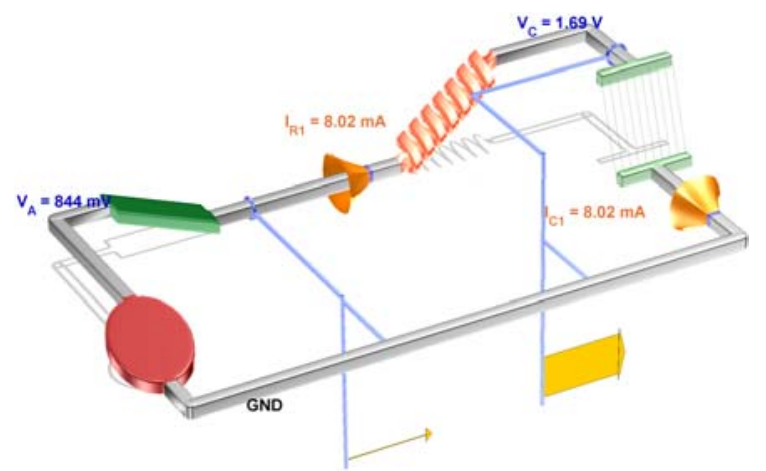

\author{
Vidéomodèle \\ Fichier AVI \\ 25 images \\ compression RLE8 \\ Format 504X336 \\ $\underline{450 \mathrm{Ko}}$
}

fig 10 : Circuit RLC série à la résonnance + puissance

\title{
4. MESURE DE PUISSANCE
}

La figure 10 constitue également une tentative de représenter la puissance instantanée consommée par un dipôle. Les sondes de tension et courant sont connectées au circuit et le produit tension (vertical) courant (horizontal) donne le "rectangle" transformé en flèche dont la pointe indique le sens de transfert de la puissance instantanée. La flèche est jaune-beige pour un échange de gauche à droite (puissance positive) et bleue dans l'autre cas. Elle évolue et change de couleur en fonction du temps et de la nature du dipôle (résistif, capacitif, inductif).

\section{CONCLUSION}

Le premier objectif était de disposer de films de qualité pour la projection en auditoire. Comme les autres personnes [1], plus de 4 étudiants sur 5 estiment que les vidéomodèles accroissent sensiblement leur compréhension intuitive des circuits et des composants. La qualité des images et l'effet dynamique permettent non seulement de voir le fonctionnement global, y compris de circuits complexes, mais également de voir des détails qui demeuraient inaccessibles pour la plupart. Les cours d'électronique de l'ECAM se donnent désormais avec ce support performant que l'on continue à développer. On espère pouvoir constituer progressivement une bibliothèque de vidéomodèles. Il s'agit d'étudier également la façon de rendre ceux-ci accessibles en permanence aux étudiants.

\section{Bibliographie}

1. F. Gueuning, Vidéomodèles - Méthode de visualisation 3D des circuits électriques en fonctionnement, présenté également à ce colloque

2. A. del Río \& D. Valdés, Three-Dimensional Model for Analog Circuit Instruction, IEEE, 1997

http://www.ewh.ieee.org/soc/es/Nov1997/07/index.htm

3. Charles R. Sullivan, Three-Dimensional Circuit Animation Visualizes Voltage, Proceedings of the 2002 American Society for Engineering Education Annual Conference \& Exposition

http://engineering.dartmouth.edu/other/3Dcircuits

/background.html 